\title{
Colorectal polyp characterization: Is my computer better than me?
}

다 (i)

\author{
Authors \\ Roshan Patel ${ }^{1}$, Bill Scuba $^{2}$, Roy Soetikno ${ }^{3}$, Tonya Kaltenbach ${ }^{1,3}$ \\ Institutions \\ 1 University of California, San Francisco, California, United \\ States \\ 2 University of Utah, Salt Lake City, Utah, United States \\ 3 San Francisco Veterans Affairs Healthcare System, San \\ Francisco, California, United States \\ Bibliography \\ DOI https://doi.org/10.1055/s-0043-124967 | \\ Endoscopy International Open 2018; 06: E279-E280 \\ (c) Georg Thieme Verlag KG Stuttgart · New York \\ ISSN 2364-3722 \\ Corresponding author \\ Tonya Kaltenbach, University of California, San Francisco - \\ VA San Francisco, 4150 Clement Street, San Francisco, CA \\ 94121 \\ Fax: +1-650-963-3535 \\ endoresection@me.com
}

Scientists and engineers have created computers that can play games with us [1-3]. In chess, these computers are as good, if not better, as the world class champions. Because they can be better, these computers allow us to learn from them. In turn, we become better. In medicine, similar systems allow the development of computer aided diagnosis (CAD), which provide suggested diagnosis to assist us [4-7]. In gastroenterology, preliminary studies showed the feasibility of CAD for diagnosing colorectal lesions in computed tomography colonography [4-6].

CADapplied during colonoscopy for colorectal cancer screening has great promise for detection and diagnosis of colorectal neoplasms. Nonpolypoid colorectal neoplasms and sessile serrated adenoma/polyps can be difficult to detect. Their appearance is subtly different than that of normal surrounding mucosa. They may be slightly red or they may have an overlying mucous cap. In addition, optical diagnosis of these nonpolyoid lesions can be less precise. Precision, on the other hand, is highly desirable as it has the potential to improve our management of polyps found at colonoscopy [8-10] because optical diagnosis can improve efficiency and reduce overall cost of screening colonoscopy. High precision can lead the practice of leaving in situ diminutive hyperplastic polyps of the rectosigmoid colon, resecting diminutive adenomas without histologic evaluation and referring polyps with submucosal invasion or advanced cancer to surgery. CAD can use NarrowBand Imaging International Colorectal Endoscopic (NICE) classification using narrow-band imaging (NBI) with or without magnification [11].

A CAD system is essentially a math equation with many variable parameters. Through a process of cleverly directed trial and error, the parameters are adjusted based on training images, where a group of correctly identified images are presented. The changes that improve the equation's ability to correctly categorize an image are kept and those that reduce its ability are discarded until no more improvements can be made.

In a recent pilot study, Tamai and coauthors assessed the feasibility of CADfor optical diagnosis of colorectal lesions [12]. The team developed a CAD system using magnifying narrow-band images to classify identified polyps as hyperplastic, adenoma, superficial submucosal, and deep submucosal lesions. The software incorporated modified NBI still polyp images to provide qualitative and quantitative characteristics. For the modified images, an experienced endoscopist determined the region of interest from the original still image, converted the NBI image into grayscale, maintained only the vessel color, and removed isolated points and holes. The software then employed Sano's colorectal magnification NBI classification. The group studied the optical diagnosis performance of CAD in 121 NBI still polyp images (21 hyperplastic, 80 adenoma/superficial submucosal invasion and 20 deep submucosal invasion). The concordance rate of optical diagnosis of the images was $90.9 \%$ between CAD and experienced endoscopists. The methods are limited, such as sample size, training and test set, and the application is for still images. Nevertheless, the results of the study showed the potential of CAD in management of colorectal neoplasms.

While Dr. Tamai and colleagues showed the potential application of CADfor polyp characterization, one could foresee CAD's potential in detection of colorectal lesions as well. Prototypes have been developed to aid detection of colorectal le- 
sions in which the systems would alert an endoscopist about subtle change that needs further investigation [13].

Endoscopists will not be replaced by CAD. Instead, we are a key component in the performance of the CAD system. Without an endoscopist to correctly identify appropriate training images, the machine learning system cannot learn and cannot perform - it has no context, no way to fix improperly identified images, and no ability to do anything on its own. To perform a colonoscopy, our very extensive knowledge base will still be needed. There are many steps before a lesion needs interpretation. We decide if the patients are suitable for the procedure. We prescribe the appropriate medications based on various considerations. We insert the colonoscope. We have to inspect the surface area of the colon and navigate barriers, such as folds, turns, and stool. We may wash or suction fluids. A significant amount of cognitive and technical work to reach the point to detect and then interpret a polyp is undertaken before CAD can be used. Suggestions by CAD will need to be interpreted in the appropriate clinical settings.

CAD has the potential to significantly change the way we perform endoscopy in the future. Coupling the power of machines with human knowledge and technique will be complementary to our mission to detect, diagnose and remove colorectal lesions, particularly flat lesions [14]. Ultimately, computers may facilitate improvement in colonoscopy performance and help us in our mission to prevent colorectal cancer incidence and mortality.

\section{Competing interests}

Dr. Kaltenbach is a consultant for Olympus America. Roy Soetikno - Consultant, Olympus America.

\section{References}

[1] "The official site of the matches - The 1997 game 4". IBM Research; http://www-03.ibm.com/ibm/history/ibm100/us/en/icons/deepblue/Deep Blue Retrieved 2017-08-01

[2] Markoff J. Computer Wins on 'Jeopardy!': Trivial, It's NotNew York Times; 16.02 2011: Retrieved from http://www.nytimes.com/2011/ 02/17/science/17jeopardywatson.html?pagewanted=all\# 2017-0801

[3] Mozur P. Google's A.I. Program Rattles Chinese Go Master as It Wins Match. New York Times; 25.05 2017: Retrieved from https://www. nytimes.com/2017/05/25/business/google-alphago-defeats-go-kejie-again.html 2017-08-01

[4] McKenna MT, Wang S, Nguyen TB et al. Strategies for improved interpretation of computer-aided detections for $\mathrm{CT}$ colonography utilizing distributed human intelligence. Medical Image Analysis 2012; 16: $1280-1292$

[5] Plumb AA, Fanshawe TR, Phillips P et al. Small Polyps at Endoluminal CT Colonography Are Often Seen But Ignored by Radiologists. AJR Am J Roentgenol 2015; 205: W424-431

[6] Tulum G, Bolat B, Osman O. A CAD of fully automated colonic polyp detection for contrasted and non-contrasted CT scans. Int J CARS 2017; $12: 627-644$

[7] Wang H, Liang Z, Li LC et al. An adaptive paradigm for computer-aided detection of colonic polyps. Phys Med Biol 2015; 60: 7207-7228

[8] Rex DK, Kahi C, O'Brien M et al. The American Society for Gastrointestinal Endoscopy PIVI (Preservation and Incorporation of Valuable Endoscopic Innovations) on real-time endoscopic assessment of the histology of diminutive colorectal polyps. Gastrointest Endosc 2011; 73: $419-422$

[9] McGill SK, Evangelou E, loannidis JP et al. Narrow band imaging to differentiate neoplastic and non-neoplastic colorectal polyps in real time: a meta-analysis of diagnostic operating characteristics. Gut 2013; 62: $1704-1713$

[10] Committee AT, Abu Dayyeh BK, Thosani N et al. ASGE Technology Committee systematic review and meta-analysis assessing the ASGE PIVI thresholds for adopting real-time endoscopic assessment of the histology of diminutive colorectal polyps. Gastrointest Endosc 2015; 81: 502 e1-e16

[11] Hewett DG, Kaltenbach T, Sano Y et al. Validation of a simple classification system for endoscopic diagnosis of small colorectal polyps using narrow-band imaging. Gastroenterology 2012; 143: 599-607 e1

[12] Tamai Naoto, Saito Yutaka, Sakamoto Taku et al. Effectiveness of computer-aided diagnosis of colorectal lesions using novel software for magnifying narrow-band imaging: a pilot study. Endosc Int Open 2017; 05: E690-E694

[13] Misawa M, Kudo S-E, Mori Y et al. Accuracy of computer-aided diagnosis based on narrow-band imaging endocytoscopy for diagnosing colorectal lesions: comparison with experts. Int J CARS 2017; 12: $757-766$

[14] Soetikno RM, Kaltenbach T, Rouse RV et al. Prevalence of nonpolypoid (flat and depressed) colorectal neoplasms in asymptomatic and symptomatic adults. JAMA 2008; 299: 1027 - 1035 